\title{
Video Article \\ Establishment and Analysis of Tumor Slice Explants As a Prerequisite for Diagnostic Testing
}

\author{
Ashwini S. Nagaraj ${ }^{1}$, Jie Bao ${ }^{1}$, Annabrita Hemmes ${ }^{1}$, Mafalda Machado ${ }^{1}$, Katja Närhi ${ }^{1}$, Emmy W. Verschuren ${ }^{1}$ \\ ${ }^{1}$ Institute for Molecular Medicine Finland (FIMM), HiLIFE, University of Helsinki
}

Correspondence to: Emmy W. Verschuren at emmy.verschuren@helsinki.fi

URL: https://www.jove.com/video/58569

DOI: doi:10.3791/58569

Keywords: Immunology and Infection, Issue 141, Organotypic explants, tissue slices, diagnostics, preclinical models, non-small cell lung cancer, rotating incubation unit, drug treatment, vibratome, KRAS, adenocarcinoma

Date Published: $11 / 29 / 2018$

Citation: Nagaraj, A.S., Bao, J., Hemmes, A., Machado, M., Närhi, K., Verschuren, E.W. Establishment and Analysis of Tumor Slice Explants As a Prerequisite for Diagnostic Testing. J. Vis. Exp. (141), e58569, doi:10.3791/58569 (2018).

\section{Abstract}

Organotypic primary tissue explant cultures, which include precision-cut slices, represent the three-dimensional (3-D) tissue architecture as well as the multicellular interactions of native tissue. Tissue slices immediately cut from freshly resected tumors preserve spatial aspects of intratumor heterogeneity (ITH), thus making them useful surrogates of in vivo biology. Careful optimization of tissue slice preparation and cultivation conditions is fundamental for the predictive diagnostic potential of tumor slice explants. In this regard, murine models are valuable, as these provide a consistent flow of tumor material to perform replicate and reproducible experiments. This protocol describes the culturing of murine lung tumor-derived tissue slices using a rotating incubation unit, a system that enables intermittent exposure of tissues to oxygen and nutrients. Our previous work showed that the use of rotating incubation units improves the viability of tissue compared to other culture methods, particularly floating slices and stagnant filter supports. Here, we further show that slice thickness influences the viability of cultured slices, suggesting that optimization of slice thickness should be done for different tissue types. Pronounced ITH in relevant oncogenic functions, such as signaling activities, stromal cell infiltration or expression of differentiation markers, necessitates evaluation of adjacent tissue slices for the expression of markers altered by drug treatment or cultivation itself. In summary, this protocol describes the generation of murine lung tumor slices and their culture on a rotating incubation unit and demonstrates how slices should be systematically analyzed for the expression of heterogeneous tissue markers, as a prerequisite prior to drug response studies.

\section{Video Link}

The video component of this article can be found at https://www.jove.com/video/58569/

\section{Introduction}

Solid tumor tissues, including lung cancer, exhibit genetic and phenotypic heterogeneity, and harbor complex microenvironments ${ }^{1,2}$. The interplay between tumor cells and their surrounding microenvironment influence on drug sensitivity and resistance mechanisms ${ }^{3}$. This highlights the need for preclinical models that can accurately model biological complexities and functions acting in native tumors. Precision-cut slices immediately derived from fresh tumors provide a unique resource, as they have a principal capacity to represent in vivo biology, at least for a short window of time, including phenotypes spatially distributed in an individual tumor. Resected clinical tumors are one of the few personalized specimens that can be obtained from a cancer patient, and their diagnostic use deserves scrutiny.

The history of organotypic cultures dates back to the early $19^{\text {th }}$ century, when human intracranial tumors were hand-cut into tissue pieces and cultured using the so-called hanging drop method. Tissue fragments were attached to a coverslip and allowed to dip into heparinized human plasma, after which the coverslips were inverted, sealed and cultured for several weeks ${ }^{4}$. Manually cut tumor pieces have since been cultured using a variety of other methods, such as on plasma clots ${ }^{5}$, in liquid media ${ }^{6}$, or on $0.45 \mu \mathrm{m}$ pore size filters ${ }^{6}$. The term "organotypic" was first used in 1954, in a study on retinal differentiation of the chick embryo eye ${ }^{7}$. This was followed by studies that used lung and heart tissue explants derived from chick embryos ${ }^{8}$, and brain explants from adult rats ${ }^{9}$.

Various slicing methods have been described, namely manual choppers ${ }^{10,11}$, the Krumdieck tissue slicer ${ }^{12,13}$, and vibratomes ${ }^{11,14,15,16}$. The Krumdieck tissue slicer generates cylindrical tissue cores, which are then sliced into circular tissue slices using a microtome. A vibratome, on the other hand, uses a vibrating blade microtome. In a study on liver slices, it was shown that the Leica vibratome generates more reproducible and consistent slices compared with the Krumdieck slicer ${ }^{15}$. Slice thicknesses ranging from 250 to $500 \mu \mathrm{m}$ have been used, and studies report maintenance of viability and morphological features even until 16 days ${ }^{14,10,17,18}$. However, tumors have variable metabolic profiles that can affect nutrient requirements, and parameters such as tissue stiffness and matrix composition can influence on aeration and nutrient flow. It is therefore likely that each tissue type requires optimization of slicing and culture conditions.

Different cultivation methods have been used to support slice cultures: i) stationary interphase culture, also called stagnant support culture, in which slices are placed on top of a semi-porous membrane insert immersed in the culture medium. In this, the top of the slices is exposed to the air, while the bottom is supplemented with nutrients via the porous insert ${ }^{14,19}$; ii) the Trowel method, originally developed to culture whole 
organs or embryonic tissue slices. In this, slices are placed on top of a cotton sheet or filter supported by a metal grid, and the filter is soaked in the culture medium. To keep the tissues moist, a thin layer of medium is added on top of the slices ${ }^{20,21,22}$. These first two are so called airliquid interphase cultures; iii) roller tube cultures, in which slices are placed inside the flat side of a plastic tube containing medium, and slow tube rotation ensures that the tissue is covered with medium during the first part of a cycle, or aerated during the second ${ }^{23}$; iv) rotating incubation units, in which slices are intermittently exposed to medium with nutrients and aeration. Different from roller tubes, in this method slices are placed on top of porous titanium grids placed in 6-well plates with culture medium ${ }^{24}$.

Tissue slices derived from resected solid tumors logically present an attractive ex vivo model in which to test the treatment response of anticancer agents, as they permit the evaluation of viability, targeted pathway activity, and molecular profiles of a specific tumor in the presence of its native tumor microenvironment. However, to evaluate whether the drug responses measured in tumor slices are predictive of in situ responses, it is important to assess to what extent tissue slices preserve tumor-specific biological functions such as cell proliferation, histopathology-specific cell differentiation or oncogenic signaling activities. The impact of mechanical stress elicited during slice preparation, slice handling, or cultureinduced adaptions on both the quality and biological functions of tissue slices are fundamental questions, tightly linked to the ability to implement tumor-derived slices for functional diagnostics.

Our IMI-funded consortium project PREDECT (http://www.predect.eu) set out to systematically address these fundamental questions, by studying slice explants from a variety of sources. Using slices derived from breast, prostate and lung cancer models, this joint effort utilized qualitative read-outs as well as quantitative hematoxylin and eosin $(\mathrm{H} \& \mathrm{E})$ - based readouts to demonstrate a requirement for atmospheric oxygen and stagnant filter supports to sustain the viability of cultured slices until $72 \mathrm{~h}$. Furthermore, immunohistochemistry (IHC) analyses on cultured slices revealed intra-slice viability gradients, evidenced as necrosis gradients in slices derived from murine non-small cell lung cancer (NSCLC), estrogen receptor (ER), HIF1 $\alpha$ and $\mathrm{yH} 2 \mathrm{AX}$ gradients in breast cancer slices, or androgen receptor (AR) expression gradients in prostate cancer slices ${ }^{25}$. Interestingly, intra-slice viability gradients in $24 \mathrm{~h}$ cultures of murine NSCLC were rescued by cultivation in a rotating incubation unit, and our recent study showed that viability was extended to $72 \mathrm{~h}^{26}$. Particularly the top side remained most viable ${ }^{26}$, endorsing that drug response analyses on slices are best carried out on this side of the tissue.

Even though it remains a question as to how far tissue slices can recapitulate in situ tumor functions, they have been extensively used to test responses to anti-cancer agents, including targeted drugs, monoclonal antibodies and chemotherapy agents ${ }^{10,11,13,14,18,27}$. We recently showed that murine NSCLC slices show dynamic changes in proliferation and oncogenic signaling activities following cultivation when compared to freshly cut $0 \mathrm{~h}$ slices ${ }^{26}$. This indicates that it is important to investigate whether the targeted in situ biological functions are appropriately preserved during cultivation, prior to perturbation studies. Despite these findings, we showed that tumor slices can model spatial response to targeted therapies, midst drug treatments remain brief $(24 \mathrm{~h})$ and are initiated at the onset of culturing ${ }^{26}$. The following protocol describes important validation aspects relevant to the establishment and analysis of tumor slice cultures, prior to their application in pharmacological drug testing.

\section{Protocol}

All mouse experiments described in this study were performed by following the guidelines from the Finnish National Board of Animal Experimentation, and were approved by the Experimental Animal Committee of the University of Helsinki and the State Provincial Office of Southern Finland (License number ESAVI/9752/04.10.07/2015).

\section{Preparations Prior to Slicing}

1. Keep the following materials ready: vibratome specimen holder, vibratome buffer tray, vibratome ice bath, $10 \mathrm{~cm}$ culture plate, 24 -well plate, $10 \mathrm{~mL}$ pipette, pipette boy, waste bag, $70 \% \mathrm{EtOH}$ in a $50 \mathrm{~mL}$ tube to disinfect the instruments, and tissue glue.

2. Prepare the vibratome: wipe the blade holder with $70 \% \mathrm{EtOH}$, attach a new blade, and perform a vibrocheck according to instructions provided in the manual (http://photos.labwrench.com/equipmentManuals/10103-3895.pdf). NOTE: It is important to perform the vibrocheck step, as it minimizes the vertical deflection of the blade and ensures good quality slices.

3. Fill each well of the 24-well plate with $1 \mathrm{~mL}$ of Hanks Balanced Salt Solution (HBSS) supplemented with $100 \mathrm{U} / \mathrm{mL}$ penicillin and $100 \mu \mathrm{g} / \mathrm{mL}$ streptomycin (HBSS + P/S); keep the plate on ice.

4. Prepare F12 culture medium supplemented with $100 \mathrm{U} / \mathrm{mL}$ penicillin, $100 \mu \mathrm{g} / \mathrm{mL}$ streptomycin, $2 \mathrm{mM}$ glutamax, $22 \mathrm{mM}$ glucose, and $10 \%$ fetal bovine serum (FBS).

NOTE: Tumor slice culture medium and growth factor supplements can vary depending on the tumor tissue ${ }^{25}$.

5. Prepare the required amount of F12 medium for drug treatment, using the same composition as described in step 1.4, but omitting the FBS NOTE: Since growth factors in the serum may affect oncogenic signaling in cultured slices, serum-free medium is recommended for shortterm drug perturbation studies on tumor slices. If longer-term slice cultures are analyzed, it is important to first evaluate the effect of serumfree medium on tissue viability and tumor-specific marker expression in untreated slice cultures.

\section{Collection of Tumor-bearing Lungs}

1. Euthanize a tumor-bearing mouse by cervical dislocation when it shows symptoms of labored breathing and loss of body weight. NOTE: $\mathrm{CO}_{2}$-mediated euthanasia is known to induce hypoxic conditions in the lungs, which may have an effect on slice viability or oncogenic activities via eliciting a hypoxic response.

2. Stretch the euthanized mouse onto a Styrofoam lid by inserting $25 \mathrm{G}$ needles in all four paws, so that the chest is exposed. Spray $70 \%$ EtOH on the surface of the exposed skin.

3. Cut open the skin from the abdomen towards the chest, and up to the neck region. Stretch the skin on either side using forceps, and insert $25 \mathrm{G}$ needles. Cut open the abdominal region, followed by the rib cage and diaphragm, to expose the lung and heart. Expose the trachea by cutting away the surrounding tissue. Keep the scissors in an angled position to avoid tissue damage. 
4. Dissect the tumor-bearing lungs together with the heart and place them into a $50 \mathrm{~mL}$ tube containing $30 \mathrm{~mL}$ of ice-cold HBSS $+\mathrm{P} / \mathrm{S}$; keep the tube ice-cold and proceed to the next step as quickly as possible.

NOTE: Delays in processing of lung tumors may alter oncogenic functions, for example signaling pathway activities.

\section{Generation of Precision-cut Lung Tumor Slices}

1. Transfer the tumor-bearing lungs in HBSS $+\mathrm{P} / \mathrm{S}$ into a $10 \mathrm{~cm}$ tissue culture plate. Separate the lung lobes using sterile scissors and forceps, and select lobes with tumors on the surface for slicing.

NOTE: Tumors $>3 \mathrm{~mm}$ in size are suitable for slicing. Since normal lung tissue surrounding the big tumor compromises the slicing due to differences in tissue stiffness, tumor tissue undergoing slicing should be separated away from the normal tissue, such that a cleared tumor region faces the vibratome blade.

2. Place the selected lung lobe with a tumor tissue on a piece of filter paper to avoid slipping, and cut a part of the normal lung or additional tumor tissue that surrounds the tumor away with a sterile scalpel to generate a flat tissue piece surface.

1. Dip the flat slide in a drop of cyanoacrylate adhesive. Mount this side onto the vibratome's specimen holder so that the tumor faces the blade in an upright position. Let the glue dry for 2-3 min.

NOTE: The normal lung tissue glued to the specimen holder does not interfere with tumor tissue slicing, and slicing is stopped before the normal tissue is reached. The tumor tissue sometimes bends due to the spongy texture of the normal lung tissue glued to the specimen holder, compromising its upright position. If this happens, glue a piece of additional normal lung support tissue next to the pre-mounted normal lung tissue to retain the tumor in an upright position (Figure 1A iii).

3. Place the specimen holder into the metal buffer tray and fill it with cold HBSS + P/S until the tissue is immersed in the buffer, and cover the buffer tray with the plexiglass lid that is provided with the instrument. Place the metal buffer tray onto the white ice bath and add ice so to keep the tissue cool while slicing.

4. Attach the white ice bath to the vibratome. Select suitable slicing settings: amplitude ranging between 2.5-2.8, slicing speed between $0.10-0.14 \mathrm{~ms}$, and a cutting thickness ranging between $160-250 \mu \mathrm{m}$. Bring the vibratome blade to the slicing position, set the slicing window and start slicing.

NOTE: Slicing settings need to be adjusted according to the hardness of the tissue. Hard tissue is easier to slice than softer tissue, and softer tissues requires slicing with lower speed $(0.1-0.12 \mathrm{~ms})$ and higher amplitude (2.6-2.8). A 4-5 mm large tumor typically provides 15-20 slices of $200 \mu \mathrm{M}$ thickness. For short-term cultivation, murine tumors can be sliced under semi-sterile conditions outside the laminar hood. However clinical tumors should always be sliced inside a class II biosafety laminar hood to avoid exposure to possible infectious agents in the human tissue.

5. Using sterile forceps, collect the slices in $1 \mathrm{~mL}$ of HBSS in separate wells of a 24-well plate held on ice, closely keeping track of the order in which slices are sliced. Mark each well of the 24-well plate according to the experimental plan. For example, mark sequential wells of a 24well plate as culture time points or as $0 \mathrm{~h}$, vehicle control $(\mathrm{C})$, drug treated (T) (Figure 1B ii).

NOTE: Do not disturb or pull the tumor tissue while collecting a slice, as this will alter the tumor's orientation with respect to the angle of the blade, leading to inconsistencies in the thicknesses of the subsequent slices.

6. When all slices are collected, transfer them onto titanium grids (2-3 slices per grid) placed in a 6 -well plate containing $2.5 \mathrm{~mL}$ of culture medium per well. Make sure that no air bubbles are formed between the titanium grid and the medium.

1. To load a slice on to the grid, keep the 6-well plate in an angled position so that a portion of the medium covers the grid, and place the slice in the medium on the grid; use forceps to spread the slice if it curls. Load the 6-well plates onto the rotating incubation unit placed inside a humidified incubator maintained at $37^{\circ} \mathrm{C}$ with $95 \%$ air and $5 \% \mathrm{CO}_{2}$, and start the rotation cycle (Figure 1C i-ii).

NOTE: Metallic grids and 6-well plates need to be accurately weight balanced before turning on the rotating unit. It is important to position the slices in the middle of the grid so that they fully alternate between the air and liquid phases during the rotation cycles. Slices that are placed too low or high are not appropriately exposed to oxygen or nutrients (Figure 1C i), which can compromise tissue viability. It is important to follow the position of the slice on the grid during cultivation, as the slice can occasionally slide down. If this happens within 1-2 h of culture onset, correct its position and note down that this sample may be damaged. Slices that are mispositioned for an extended period of time should be discarded, as tissue viability is significantly affected by improper oxygenation and nutrient supply.

7. Collect a tissue slice adjacent to the cultured slices as a $0 \mathrm{~h}$, uncultured, reference. Collect at least three reference slices to represent the top, middle and center of the tissue being sliced. Fix the $0 \mathrm{~h}$ slices immediately and process as described in 5.1 .

NOTE: If the number of slices is limited, e.g., if multiple compound treatments or technical replicates are done, comparisons of each treated sample with its neighboring $0 \mathrm{~h}$ sample can be difficult. In such cases, use the nearest $0 \mathrm{~h}$ slice (at least $400-600 \mu \mathrm{m}$ apart) to assess relative tissue viability or expression of relevant markers at culture onset.

8. For long-term cultivation, replenish the culture medium every day. Lift the grid containing the tissue slices using sterile forceps, and place it in an empty well of the 6 -well plate; replace $70 \%$ of the medium with fresh culture medium, and place the grid back in the medium. Continue the rotation cycle as explained in step 3.6.

\section{Treatment of Tumor Slices with Small Molecule Inhibitors}

1. Prepare the required concentrations of compounds in treatment medium. Typically, a 10-fold higher drug concentration compared to the IC50s measured in cell cultures is required to achieve target inhibition in tissue slices. To avoid unspecific cytotoxicity, test a range of concentrations to obtain minimally effective concentration for each compound. Here, $0.1-1 \mu \mathrm{M}$ of the PI3K/mTOR inhibitor dactolisib and $0.05-0.5 \mu \mathrm{M}$ of the MEK inhibitor selumetinib were tested on murine NSCLC slices.

2. Add $2.5 \mathrm{~mL}$ of media with the diluted drug or DMSO or other vehicle control into the 6 -well plate. Place the titanium grids into the wells.

3. Place the tissue slices onto the grids as described in step 3.6.

4. Perform vehicle or drug treatments for $24 \mathrm{~h}$. Proceed with tissue fixation and processing of the slices into paraffin blocks as described in section 5 . 
NOTE: Duration of drug treatment can be optimized depending on the objective of the experiment, taking into consideration the ability of tissue slices to retain in situ tissue functions analyzed during the culture period.

\section{Fixation and Processing of the Tissue Slices}

1. Carefully lift the uncultured $0 \mathrm{~h}$ reference or cultured slice onto a filter paper soaked in PBS.

1. To do this, add $2-3 \mathrm{~mL}$ of PBS on top of a filter paper placed in a $10 \mathrm{~cm}$ plate. Place the slice on top of the filter paper and lift out the filter paper using a pair of forceps.

2. Transfer the filter paper into a histocassette, and add a drop of diluted hematoxylin (1:1 in deionized water) on top of the tissue slice to visibly mark the position of the slice during the subsequent processing steps (Figure 1D).

3. Close the cassette, and transfer it into $4 \%$ neutral buffered formalin solution. Fix the tissues overnight at $4{ }^{\circ} \mathrm{C}$.

NOTE: While placing the slice on top of the filter paper, make sure that the top section of the slice is facing upwards; this is necessary to follow the top, middle, and bottom section of a slice during sectioning and analysis as described in step 6.3.

2. The next day, transfer the cassettes into $70 \% \mathrm{EtOH}$, and immediately proceed with the paraffin-embedding tissue processing step.

3. Prior to tissue processing, wash the histocasettes in $100 \% \mathrm{EtOH} 2 \mathrm{x}$ for $10 \mathrm{~min}$ each. In this case, use a microwave station for tissue processing. Select the program used for $1 \mathrm{~mm}$ tissue thickness and follow the instructions provided in the manual (https:// www.totaltissuediagnostics.com/images/MM073-005_-_KOS_Operator_Manual.pdf).

NOTE: When using other tissue processing machines, use the program suitable for thin tissue samples.

4. For paraffin-embedding, open a histocassette and use a scalpel to carefully lift the slice from the filter paper. Discard the filter paper, and transfer the slice into a mold containing liquid paraffin.

1. Press the tissue against the bottom of the embedding mold, for instance with a flat weight, to ensure even sectioning. Place the bottom part of the histocasette on top of the mold, add liquid paraffin on top of it. Let the mold cool on a cold pate for 30 min, and separate the mold from the paraffin block.

NOTE: As an alternative to horizontal embedding, it is possible to embed the tumor slice vertically by positioning the slice in an upright position. Vertical sections readily permit analysis of gradients in viability or functional marker expression on a single section ${ }^{25}$. Gradient analysis with horizontally-embedded slices requires paraffin sectioning as described in step 6.2 .

\section{Processing and Analysis of Formalin-fixed and Paraffin-embedded (FFPE) Tissues}

1. Prepare $4 \mu \mathrm{m}$ thin sections of the FFPE tissue slice blocks using a microtome. When sectioning, adjust the angle of the block so that the surface of the block is horizontally oriented with respect to the blade; this is necessary to obtain even sections throughout the tissue.

2. To enable capture of a potential culture-induced viability gradient, cell migration across the slices, or gradients in biomarker expression across cultured slices, collect sections from the top, center and bottom layers of each of the tissue slice on object slides as explained below.

3. Collect sequential tissue sections of the paraffin-embedded tissue slice first on the upper part of the glass slides. Continue collecting the sections of the deeper tissue layers to the middle followed by bottom part of the glass slides (Figure 1E). NOTE: To accommodate three sections on a glass slide, trim away the excess of paraffin surrounding the embedded tissue slice.

4. Allow the sections to dry overnight at $37^{\circ} \mathrm{C}$, and proceed with $\mathrm{H} \& \mathrm{E}$ staining or immunohistochemistry as described below. NOTE: Loss of antigenicity can occur when FFPE sections are stored at high temperatures or for extended periods of time. Paraffin sections are recommended to be stored at $4{ }^{\circ} \mathrm{C}$, and IHC analyses should be carried out within 6 months after sectioning.

1. For H\&E staining, deparaffinize and rehydrate the paraffin sections as follows: xylene $3 x$ for $5 \mathrm{~min}, 100 \%$ EtOH $3 x$ for $1 \mathrm{~min}, 96 \%$ EtOH $2 \mathrm{x}$ for $1 \mathrm{~min}, 70 \%$ EtOH $1 \times 1 \mathrm{~min}$, and deionized water $2 \mathrm{x}$ for $1 \mathrm{~min}$.

2. Incubate the sections in freshly filtered hematoxylin solution for $10 \mathrm{~min}$, and wash under running tap water for $5 \mathrm{~min}$. Dip the sections in acid alcohol $(1 \% \mathrm{HCl}$ in $70 \% \mathrm{EtOH})$ for 2 times, and wash under running tap water for 5 min followed by incubation with $0.5 \%$ eosin for 2 min.

3. Following the eosin step, dehydrate the sections by immersing the slides in alcohol and xylene solutions, as follows: $96 \%$ EtOH $2 x$ for $15 \mathrm{~s}, 100 \% \mathrm{EtOH} 3 \mathrm{x}$ for $30 \mathrm{~s}$, xylene $3 \mathrm{x}$ for $1 \mathrm{~min}$. Finally, embed the sections in a xylene-based mounting medium.

\section{Analysis of Tissue Viability and Biomarker Expression}

1. Acquire high resolution images by generating whole slide scans of H\&E-stained slides using a scanner. To assess tissue viability, take snapshots from the tissue scans representing the top, middle and bottom sections of the slices.

1. Using photo manipulator software, manually draw masks on the necrotic areas of the tissues, followed by quantification of necrotic regions using MATLAB.

2. Calculate the relative viability of the slices cultivated for different time points compared to the nearest $0 \mathrm{~h}$ slice as done in our previous study (Närhi et al., Figure S2B and C) ${ }^{26}$. Similarly, assess potential intra-slice viability gradients by quantifying viability in the top, middle and bottom section of a cultured slice, and calculate the relative viability of each section to its closest $0 \mathrm{~h}$ slice. NOTE: While the mere cultivation of murine NSCLC slices induces necrotic cell death, biological responses to ex vivo culture conditions vary depending on the tumor tissue. For example, gradients in HIF1a, ER, and macrophages marked by F4/80 were detected in filter-supported slice cultures derived from a breast cancer model ${ }^{25}$. Therefore, H\&E- as well as IHC-based analyses of cultured slices need to be considered for each tissue type.

2. Perform IHC on the paraffin sections. The following $\mathrm{IHC}$ protocol is a starting point, and requires further optimization for other antibodies. Briefly, deparaffinize and rehydrate the paraffin sections as follows: xylene $3 x$ for $5 \mathrm{~min}, 100 \%$ EtOH $3 x$ for $1 \mathrm{~min}, 96 \%$ EtOH $2 x$ for $1 \mathrm{~min}$, $70 \%$ EtoH $1 \mathrm{x}$ for $1 \mathrm{~min}$, and deionized water $2 \mathrm{x}$ for $1 \mathrm{~min}$. 
1. To expose the antigenic epitopes, perform heat-mediated antigen retrieval using $10 \mathrm{mM}$ citric acid at pH 6 in a PT module, followed by blocking with $1 \%$ Bovine Serum Albumin (BSA) and 10\% Normal Goat Serum (NGS) in 1x PBS for 30 min at ambient temperature $\left(21-23^{\circ} \mathrm{C}\right)$.

2. Incubate the primary antibody diluted in $1 \%$ BSA and $5 \%$ NGS in $1 \times$ PBS, either for $1-2 \mathrm{~h}$ at ambient temperature or overnight at $4{ }^{\circ} \mathrm{C}$. Incubate with anti-rabbit secondary antibody, for 30 min at ambient temperature, followed by detection using 3,3'-Diaminobenzidine (DAB).

3. Sections are counterstained with hematoxylin (diluted to 1:10 in deionized water) for $30 \mathrm{~s}$, and wash under tap water for 5 min, followed by dehydration by immersing the slides in alcohol and xylene solutions, as follows: $70 \% \mathrm{EtOH} 1 \mathrm{x}, 96 \% \mathrm{EtOH} 2 \mathrm{x}, 100 \% \mathrm{EtOH} 3 \mathrm{x}$, xylene $3 x$ ( 1 min each step). Finally, embed the sections in a xylene-based mounting medium.

3. Acquire whole slide scans of IHC-stained slides, using an image viewer export them as TIFF images at a magnification that captures the whole tissue region to be analyzed, and perform the quantifications using ImageJ.

NOTE: As an alternative to the scanner, microscopic images at 20X or 40X magnification can be acquired for quantifications. Image magnification can be chosen depending on the marker to be quantified; high magnification images are recommended for nuclear staining, while cytoplasmic or membrane markers can be quantified using $20 \mathrm{X}$ images.

1. For quantification of nuclear markers, in this case NKX2-1 expression, convert each image to 16-bit images using Fiji-ImageJ, and upload images for image analysis.

2. Customize the image analysis pipeline depending on the analysis. In this protocol, use the following pipeline for the quantification of NKX2-1 positive nuclei: Identify Primary Objects | Measuring Object Intensity | Filter Objects (minimal value=0.0025) | Calculate Math. The results are represented as percentages of DAB-stained nuclei of the total number of nuclei.

\section{Representative Results}

Figure 1 represents the workflow for the generation, cultivation and analysis of precision-cut tissue slices derived from murine NSCLC tumors. For this demonstration, we utilized tumors from a genetically engineered mouse model (GEMM) harboring conditional activation of Kras ${ }^{G 12 D}$ together with the loss of $L k b 1$ (also known as Serine/Threonine Kinase 11), hereafter called KL. Mouse breeding and lung tumor initiation was performed as described $\mathrm{in}^{26,28}$. Figure $2 \mathrm{~A}$ demonstrates the effect of tissue slice thickness on the viability of slices cultured for $24 \mathrm{~h}$ using a rotating incubation unit. The results show that $160 \mu \mathrm{m}$ thin slices contain large necrotic areas across the slice. In addition, $250 \mu \mathrm{m}$ thin slices show a necrosis gradient across the slice compared to $200 \mu \mathrm{m}$ thin slices. It is likely that the poor overall viability of the thinnest $160 \mu \mathrm{m}$ is caused by technical handling during positioning of the slices on top of the grids, as these are fragile and tend to curl. On the other hand, when slices are too thick, they can become prone to deficiencies in oxygen or nutrient diffusion across the slices, which in murine NSCLC explants is evidenced as necrotic death gradient ${ }^{25}$. However, it should be noted that slices with variable thicknesses can be generated from one tumor, despite use of identical vibratome settings. It is therefore recommended to analyze multiple replicates from different tumor samples. Importantly, each tissue type requires slice thickness optimization to achieve maximum viability, as the tissue texture and hardness can affect oxygenation and nutrient flow. Figure 2B-2C illustrates quantitative IHC analyses of NKX2-1 expression, a marker of well-differentiated lung adenocarcinoma (AC) in samples cultivated up to $72 \mathrm{~h}$ and matched $0 \mathrm{~h}$ slices. Results show that NKX2-1 expression is not significantly altered in cultured slices as compared to $0 \mathrm{~h}$ uncultured slices, suggesting that the process of cultivation does not overtly affect the differentiation status of AC tumor tissue. Figure 2D demonstrates the utility of tumor tissue slices for assessing the effectiveness of targeted drugs. We recently showed that Kras mutant murine ACs exhibit high expression of phosphorylated ERK1/2 (marking increased MAPK pathway activity) when compared to adenosquamous (ASC) tumors, while expression of phosphorylated 4EBP1 (marking mTOR activity) is similarly expressed in both AC and ASC tumors ${ }^{26}$. To test if these pathways can be effectively targeted on tissue slices, KL AC tissue slices were treated with DMSO or titrated amounts of compounds, namely $0.1-1 \mu \mathrm{M}$ dactolisib to target the mTOR pathway or $0.05-0.5 \mu \mathrm{M}$ selumetinib to target the MAPK pathway. Results show that $1 \mu \mathrm{M}$ dactolisib or $0.5 \mu \mathrm{M}$ of selumetinib are effective in inhibiting the phosphorylation of 4EBP1 or ERK1/2, respectively. Furthermore, dose-dependent inhibition of the targeted phosphoproteins indicates that tissue slices can also be utilized to validate phosphorylation-specific antibodies. 
A

Preparation of lung tumor for slicing

i) Collect tumor-bearing lungs

ii) Dissect tumor tissue

iii) Mount on specimen holder
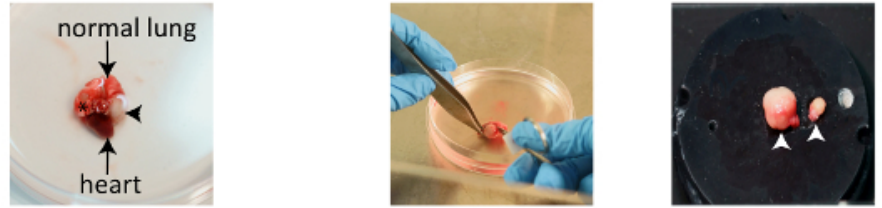

Tumor

Normal lung

specimen holder

B Vibratome sectioning and collection of tissue slices

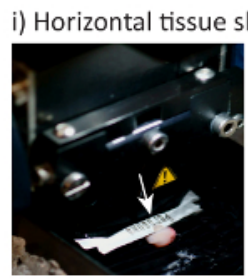

ii) Collect slices in order of slicing

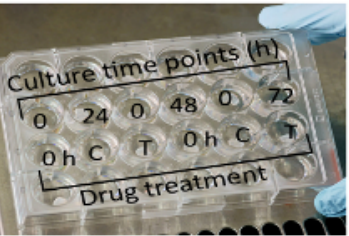

C

Placing the slices for cultivation on rotating incubation unit

i) Place slice on titanium grid

ii) Balance 6-well plates before cultivation



D

Generate FFPE blocks

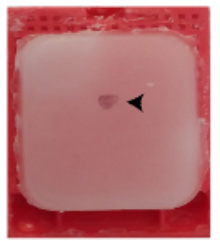

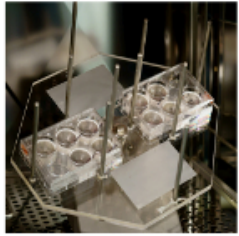

E

Analysis of the tumor tissue slices

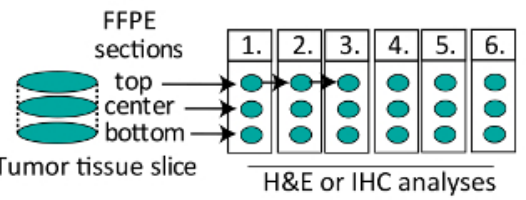

Figure 1: Schematic representation of the workflow for establishment and analysis of murine NSCLC tumor-derived slice explants. (A) Schematic describing the collection and preparation of tumor-bearing lungs for slicing. Lung lobes are harvested from a mouse and tumor tissue is dissected away from normal tissue. The black arrowhead and asterisk indicate approximately $4 \mathrm{~mm}$ and $1 \mathrm{~mm}$ tumors, respectively. The white arrowhead indicates lung tissue glued to the surface of the specimen holder. The red arrow points at an additional piece of normal lung support tissue to retain the tumor in an upright position. (B) Vibratome slicing and collection of tissue slices. White arrow indicates the slicing direction. Collection of sequential slices into a 24-well plate containing cold HBSS + P/S. The slices can either be cultured for different time points (here, 24-72 h) to assess tumor-specific marker expression during cultivation (top row), or can be used to perform drug treatments. C: vehicle control, T: drug treatment (bottom row). (C) Placing the tissue slice for cultivation using rotating incubation units. Tilt the 6-well plate so that some medium covers the top of the grid, place the tissue slice in the middle of the grid on top of the medium, and spread the slice using forceps. Ensure that the 6-well plates are weight balanced for a smooth rotation cycle. X: indicates incorrect, and $\mathscr{N}$ : indicates correct positioning of the slice. (D) Photograph of the FFPE block of a tumor slice. Black arrow points at paraffin-embedded tissue slice stained with hematoxylin. (E) Schematics showing the sectioning order of the slices in FFPE blocks; these sections can be processed to assess tissue viability and tumorspecific biomarker expression. Please click here to view a larger version of this figure. 
A

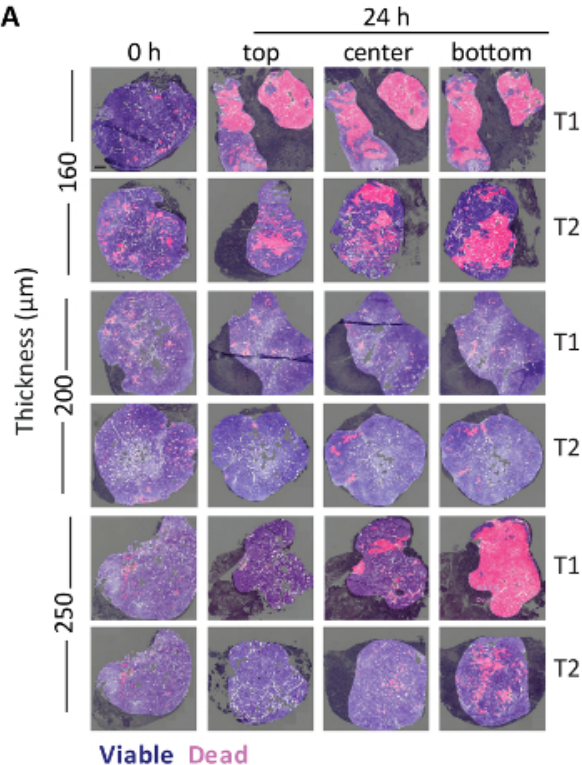

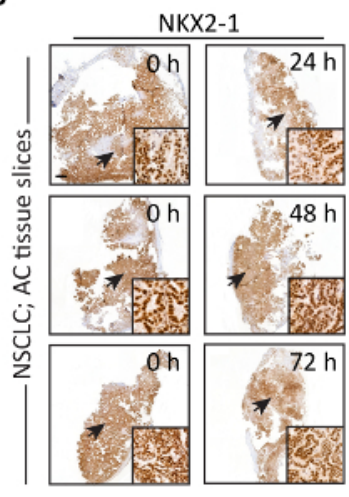

C

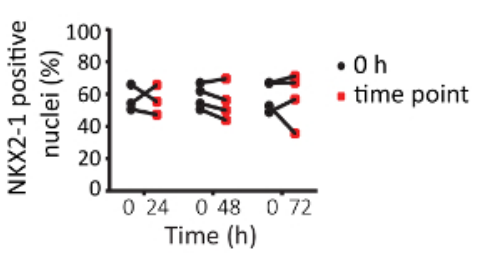

D NSCLC; AC tissue slices

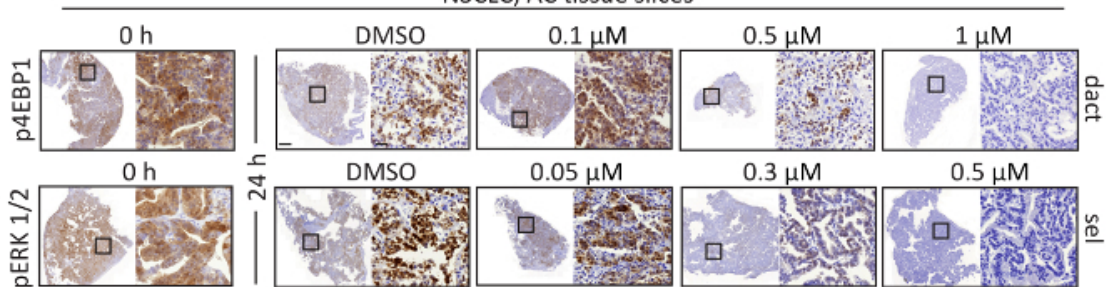

Figure 2: Assessment of viability and histotype-specific marker expression, and targeted drug treatment on NSCLC tissue slices. (A) Representative H\&E images of AC NSCLC slices of the indicated thicknesses cultured for $24 \mathrm{~h}$. $200 \mu \mathrm{m}$ thin slices maintain better viability compared to $160 \mu \mathrm{m}$ or $250 \mu \mathrm{m}$ thin slices. Dark blue represents H\&E stained viable tissue, and pink indicates pseudocolored necrotic regions. Light blue indicates regions excluded from the analysis, either due to poor tissue quality or presence of fibrous stroma. T1 and T2 represent biological replicates derived from two different tumors. Scale bar $=500 \mu \mathrm{m}$. (B) Representative IHC images of NKX2-1 expression in AC slices cultured for the indicated time points. Arrow indicates the area shown in higher magnification. Results show that NKX2-1 expression is not altered in the cultured slices compared to $0 \mathrm{~h}$ slices. Scale bar $=500 \mu \mathrm{m}$ and $50 \mu \mathrm{m}$ for low and high magnifications, respectively. (C) Quantification of the data shown in (B). (D) Representative IHC images of phosphorylated 4EBP1 or ERK1/2 expression in $0 \mathrm{~h}$ slices, or slices treated with DMSO or titrated amounts of dactolisib (dact, top row) or phosphorylated ERK1/2 expression in $0 \mathrm{~h}$ slice, or slices treated with DMSO or selumetinib (sel, bottom row). Black square boxes indicate areas shown in higher magnification. Scale bar $=1 \mathrm{~mm}$ or $50 \mu \mathrm{m}$ for low or high magnification, respectively. Please click here to view a larger version of this figure.

\section{Discussion}

Various complex in vitro tumor models, including 3D cultures and organoids, have been developed to recapitulate the architecture and oncogenic functions of in vivo tumor tissue ${ }^{29,30}$. However, the establishment of 3D cultures or organoids involves tissue dissociation and selective growth of a single cell type or co-culture of a select few cell types in an artificial environment. As a consequence, such models incompletely capture the intricacies of tumor heterogeneity and tumor-stroma interactions. Organotypic tumor slices, on the other hand, maintain the tissue architecture and biological complexities of the in situ tumor, without extensive manipulation. This ability of tissue slices to model tumor cells in their native microenvironment renders them particularly attractive for preclinical studies. We previously reported an optimized workflow for the establishment and analysis of precision-cut tumor slices, and showed that, compared to filter supports, a rotating incubation unit improve the viability of shortterm murine NSCLC slice cultures ${ }^{25,31}$. However, cultivation on rotating units is technically challenging and requires constant monitoring. We here present a protocol for tumor tissue slice generation and practical use of a rotating incubation unit to culture them, as well as accompanying methods to monitor the ability of slices to capture in situ tumor biology, a prerequisite prior to drug response testing.

Several critical steps in the protocol ensure tissue integrity and viability of the tumor slices. If normal lung tissue surrounds the tumor, the vibratome can generate slices with inconsistent thickness or damage the slices, due to differences in texture and stiffness between normal and tumor tissues. It thus is important to remove the surrounding normal lung tissue prior to tumor slicing. Another critical step is the slicing thickness, which should be carefully optimized for each tissue type. Furthermore, once sliced, it is critical that the slice is placed approximately in the middle of the grid, so to ensure accurate intermittent dipping in culture medium and oxygen exposure. Finally, it is important to follow the position of a slice during its rotation period, as a slice can drop down in to the medium; if this happens, further actions can be taken as explained in step 3.6 of the protocol. 
In addition to tissue handling to assure integrity, there are also critical steps in the IHC analysis to interpret how the slice resembles the native tissue. Our previous study showed that murine NSCLC tumors exhibit pronounced intra-tumor spatial heterogeneity in oncogenic signaling activities $^{26}$. This means that the use of spatially distinct tissue slices for controls or drug-treated samples can affect reliable experimental data interpretation, and hence closely adjacent slices should be used as controls and test samples. We further showed that while proliferation or oncogenic phosphoprotein expression in freshly cut uncultured $0 \mathrm{~h}$ slices were similar to in situ tumors, cultured slices showed altered oncogenic phosphoprotein expression, specifically altered p4EBP1 and pSRC, as well as altered proliferation analyzed by Ki67 IHC. Altered p4EBP1 expression was similarly detected in $24 \mathrm{~h}$ human NSCLC and prostate cancer slice cultures (Narhi et al., Supplementary Figure S3B-S3C, S5 and $S 7)^{26}$. These findings endorse that comparison of cultured slices with their nearest $0 \mathrm{~h}$ uncultured slice is critical to assess the preservation of in situ tumor functions in cultured slices.

Despite improving the viability of organotypic slices ${ }^{25}$, there are limitations with the rotator system in terms of technicalities. Placing the tissue slices onto titanium grids is more challenging compared to filter inserts, and a rotating incubation unit may not be available. As an alternative, stagnant filter supports can be used, but in that case only the air-exposed side of the slices should be analyzed, as air-to-filter gradients in viability and hypoxia measured by HIF1 $\alpha$ expression are rapidly formed in filter-supported slice cultures (Davies et al., Figure 5 and Figure $7 \mathrm{~A}-7 \mathrm{~B})^{25}$. We have further shown that tumor slice cultures can exhibit altered proliferation and oncogenic signaling activities compared to their native tumors ${ }^{26}$, possibly because of wound-healing responses or metabolic adaptation of the slices to ex vivo culture ${ }^{32}$. Although gross morphological features of the murine NSCLC tumors were maintained during $72 \mathrm{~h}$ cultivation, culture-induced proliferative changes may affect accurate grading of the cultivated slices. Thus, tissue slices should only be utilized for short-term functional studies.

Use of a rotating incubation unit at least partially rescues intra-slice viability or biomarker expression gradients, particularly during the first 24 $\mathrm{h}$ of culture. Once validated for integrity and function, this provides tissue material for functional studies, such as drug treatment studies. In addition to drug response profiling, altered target expression following drug treatment can also benefit antibody validation. This is particularly relevant for the detection of murine epitopes with mouse monoclonal antibodies, as these tend to give high staining background. In addition, wellvalidated antibodies are required to achieve reliable and reproducible data in diagnostic and clinical settings. Thus, modulation of the abundance or phosphorylation of relevant epitopes following drug treatment in tissues slices provides a handy practical application in antibody validation. A major advantage of tumor slice cultures is the ability to model spatially-distributed functions, including oncogenic signaling activities or drug response in tumor or stromal cells, which makes them an attractive ex vivo model. However, slices rotate during cultivation, and the process of cultivation can further damage the tissue particularly at the edges. It is therefore challenging to precisely overlay the biomarker-stained $\mathrm{IHC}$ images of $\mathrm{O}$ h slices with the necrotic regions detected in cultured slices, which compromises the ability to precisely link spatial biomarker activities to drug response. In addition, tumor-intrinsic, culture-induced and drug-induced necrotic responses are indistinguishable at least in murine NSCLC tissue slices, compromising accurate quantitation of spatial drug responses. Finally, the use of tumor slice cultures permits a researcher to the test multiple compounds on the same tumor, without a need to treat animals, thus refining, reducing, and replacing experiments on laboratory animals.

As a future application, the described protocol can be adopted to clinical solid tumor samples. Further tissue type-dependent modifications or optimizations are likely required, starting with adjustments to the vibratome settings including slice thickness and vibration speed to optimize these for tumor texture or stiffness. In addition, nutrient and growth factor requirement may vary for different tumor tissues. As an example, breast cancer slices have been cultured with insulin supplemented in the medium ${ }^{13,18}$. Given that limited tissue material is obtained during surgery or biopsy, optimization of patient-derived tumor slice cultures can be challenging due to difficulties in obtaining sufficient numbers of replicate samples. Furthermore, data reproducibility is also challenged by pronounced patient-to-patient sample heterogeneity, particularly in the percentage of tumor cells versus fibrotic regions or stromal infiltrates, as well as necrotic tissue components. Finally, application of tumor slices in diagnostic settings would require investigation of the extent to which drug responses in slice explants of pre-treatment biopsies matches to posttreatment in vivo responses.

\section{Disclosures}

Authors declare no conflict of interest.

\section{Acknowledgments}

This research work received financial support from Innovative Medicines Initiative Joint Undertaking grant agreement $n^{\circ} 115188$, the University of Helsinki Doctoral Programme in Biomedicine scholarships (A.S.N.), and the Sigrid Juselius and Orion-Farmos Foundations (E.W.V.). We sincerely thank our PREDECT tissue slice platform consortium members (www.predect.eu), namely John Hickman, Heiko van der Kuip, Meng Dong, Emma Davies, Simon Barry, Wytske van Weerden and Hanneke van Zoggel, for collaborative development of the slice technology. We thank Taija af Hällström and Siv Knaappila for support in setting up the rotator system, and Riku Turkki for support with the MATLAB analysis. Jouko Siro is thanked for capturing the Figure 1 photographs. We thank the FIMM WebMicroscope team for scanning histological slides, and the Laboratory Animal Centre for husbandry support.

\section{References}

1. Bruin, E. C., McGranahan, N., \& Swanton, C. Analysis of intratumor heterogeneity unravels lung cancer evolution. Molecular and Cellular Oncology. 2 (3), e985549 (2015).

2. Travis, W. D. et al. The 2015 World Health Organization Classification of Lung Tumors: Impact of Genetic, Clinical and Radiologic Advances Since the 2004 Classification. Journal of Thoracic Oncology. 10 (9), 1243-1260 (2015).

3. Junttila, M. R., \& de Sauvage, F. J. Influence of tumour micro-environment heterogeneity on therapeutic response. Nature. 501 (7467), 346-354 (2013). 
4. Kredel, F. E. Tissue Culture of Intracranial Tumors with a Note on the Meningiomas. The American Journal of Pathology. 4 (4), $337-340.333$ (1928).

5. Wright, J. C. et al. Further investigation of the relation between the clinical and tissue culture response to chemotherapeutic agents on human cancer. Cancer. 15 284-293 (1962).

6. Roller, M. R., Owen, S. P., \& Heidelberger, C. Studies on the organ culture of human tumors. Cancer Research. 26 (4), 626-637 (1966).

7. Reinbold, R. [Organotypic differentiation of the eye of the chick embryo in vitro]. Comptes rendus des séances de la Société de biologie et de ses filiales. 148 (15-18), 1493-1495 (1954).

8. Loffredo Sampaolo, C., \& Sampaolo, G. [Organotypic cultures of chick embryo lung; some histologic and histochemical aspects]. Bollettino della Societa Italiano di Biologia Sperimentale. 32 (7-8), 797-801 (1956).

9. Bousquet, J., \& Meunier, J. M. [Organotypic culture, on natural and artificial media, of fragments of the adult rat hypophysis]. Comptes rendus des séances de la Société de biologie et de ses filiales. 156 65-67 (1962).

10. Merz, F. et al. Organotypic slice cultures of human glioblastoma reveal different susceptibilities to treatments. Neuro-Oncology. 15 (6), $670-681$ (2013).

11. Gerlach, M. M. et al. Slice cultures from head and neck squamous cell carcinoma: a novel test system for drug susceptibility and mechanisms of resistance. The British Journal of Cancer. 110 (2), 479-488 (2014).

12. Estes, J. M. et al. Efficacy of anti-death receptor 5 (DR5) antibody (TRA-8) against primary human ovarian carcinoma using a novel ex vivo tissue slice model. Gynecologic Oncology. 105 (2), 291-298 (2007).

13. Kuip, H. et al. Short term culture of breast cancer tissues to study the activity of the anticancer drug taxol in an intact tumor environment. The British Journal of Cancer. 686 (2006).

14. Vaira, V. et al. Preclinical model of organotypic culture for pharmacodynamic profiling of human tumors. Proceedings of the National Academy of Sciences. 107 (18), 8352-8356 (2010).

15. Zimmermann, M. et al. Improved reproducibility in preparing precision-cut liver tissue slices. Cytotechnology. 61 (3), 145-152 (2009).

16. Wang, K. et al. Cardiac tissue slices: preparation, handling, and successful optical mapping. American journal of physiology. Heart and circulatory physiology. 308 (9), H1112-1125 (2015).

17. Jaamaa, S. et al. DNA damage recognition via activated ATM and p53 pathway in nonproliferating human prostate tissue. Cancer Research. 70 (21), 8630-8641 (2010).

18. Naipal, K. A. et al. Tumor slice culture system to assess drug response of primary breast cancer. The British Journal of Cancer. 1678 (2016).

19. Gahwiler, B. H., Thompson, S. M., \& Muller, D. Preparation and maintenance of organotypic slice cultures of CNS tissue. Current protocols in neuroscience. Chapter 6 Unit 6.11 (2001).

20. Alfaqeeh, S. A., \& Tucker, A. S. The slice culture method for following development of tooth germs in explant culture. Journal of Visualized Experiments. 10.3791/50824 (81), e50824 (2013).

21. Rak-Raszewska, A., Hauser, P. V., \& Vainio, S. Organ In Vitro Culture: What Have We Learned about Early Kidney Development? Stem Cells International. 2015959807 (2015).

22. Trowell, O. A. The culture of mature organs in a synthetic medium. Experimental Cell Research. 16 (1), 118-147 (1959).

23. Gahwiler, B. H., Capogna, M., Debanne, D., McKinney, R. A., \& Thompson, S. M. Organotypic slice cultures: a technique has come of age. Trends in Neurosciences. 20 (10), 471-477 (1997)

24. Kiviharju-af Hallstrom, T. M. et al. Human prostate epithelium lacks Wee1A-mediated DNA damage-induced checkpoint enforcement. Proceedings of the National Academy of Science. 104 (17), 7211-7216 (2007).

25. Davies, E. J. et al. Capturing complex tumour biology in vitro: histological and molecular characterisation of precision cut slices. Scientific Reports. 517187 (2015).

26. Narhi, K. et al. Spatial aspects of oncogenic signalling determine the response to combination therapy in slice explants from Kras-driven lung tumours. The Journal of Pathology. 245 (1), 101-113 (2018).

27. Majumder, B. et al. Predicting clinical response to anticancer drugs using an ex vivo platform that captures tumour heterogeneity. Nature Communications. 66169 (2015)

28. Nagaraj, A. S. et al. Cell of Origin Links Histotype Spectrum to Immune Microenvironment Diversity in Non-small-Cell Lung Cancer Driven by Mutant Kras and Loss of Lkb1. Cell Reports. 18 (3), 673-684 (2017).

29. Lv, D., Hu, Z., Lu, L., Lu, H., \& Xu, X. Three-dimensional cell culture: A powerful tool in tumor research and drug discovery. Oncology Letters. 14 (6), 6999-7010 (2017).

30. Weeber, F., Ooft, S. N., Dijkstra, K. K., \& Voest, E. E. Tumor Organoids as a Pre-clinical Cancer Model for Drug Discovery. Cell Chemical Biology. 24 (9), 1092-1100 (2017).

31. Hoogt, R. et al. Protocols and characterization data for 2D, 3D, and slice-based tumor models from the PREDECT project. Scientific Data. 4 170170 (2017)

32. Davidson, S. M. et al. Environment Impacts the Metabolic Dependencies of Ras-Driven Non-Small Cell Lung Cancer. Cell Metabolism. 23 (3), 517-528 (2016). 